\title{
Selective data analysis for diamond detectors in neutron fields
}

\author{
Christina Weiss $^{1, \text { a }}$, Helmut Frais-Kölbl ${ }^{2}$, Erich Griesmayer ${ }^{1}$, and Pavel Kavrigin ${ }^{1}$ \\ 1 CIVIDEC Instrumentation, Wien, Austria \\ 2 University of Applied Sciences, Wr. Neustadt, Austria
}

\begin{abstract}
Detectors based on synthetic chemical vapor deposition diamond gain importance in various neutron applications. The superior thermal robustness and the excellent radiation hardness of diamond as well as its excellent electronic properties make this material uniquely suited for rough environments, such as nuclear fission and fusion reactors. The intrinsic electronic properties of single-crystal diamond sensors allow distinguishing various interactions in the detector. This can be used to successfully suppress background of $\gamma$-rays and charged particles in different neutron experiments, such as neutron flux measurements in thermal nuclear reactors or cross-section measurements in fast neutron fields. A novel technique of distinguishing background reactions in neutron experiments with diamond detectors will be presented. A proof of principle will be given on the basis of experimental results in thermal and fast neutron fields.
\end{abstract}

\section{Introduction}

In the past years, chemical vapor deposition (CVD) diamond gained in importance as detector material in neutron applications $[1,2]$. This is due to its excellent thermal and mechanical robustness and its high radiation hardness. Due to the very good spectroscopic properties [5] and the high efficiency, single-crystal CVD (sCVD) diamond is generally the diamond detector material of choice in neutron applications. There are continuous efforts to exploit this detector material for applications in nuclear fusion technology [3], especially for $\mathrm{d}-\mathrm{d}$ and $\mathrm{d}-\mathrm{t}$ plasma reactors, as well as for applications in nuclear reactor technology [4].

All measurements in neutron fields suffer from background, such as considerable $\gamma$-flux from the neutron source or charged particles from neutron-induced nuclear reactions in surrounding materials. These background contributions often dominate the spectrum and make it difficult to extract the neutron interactions from the spectrum.

The presented technique in this paper exploits the intrinsic electronic properties of sCVD diamond sensors for the identification of different particle species. This serves as the basis for the separation of different reactions and finally for the efficient rejection of background in the experiment. When using this technique, one obtains spectra in mixed particle fields which are not accessible with conventional spectroscopic measurement techniques.

\section{Experimental requirements}

The presented analysis technique is based on the signalshape analysis of the ionization current from SCVD diamond sensors, which is often called the transientcurrent-technique (TCT). The sensor material should be of high electronic grade and have low impurities, such as less

a e-mail: christina.weiss@cividec.at than 5 ppb Nitrogen concentration and less than $0.03 \mathrm{ppb}$ nitrogen-vacancy (NV) center concentration, according to the manufacturer [6]. Low impurities are a requirement not to deteriorate the intrinsic detector signal [7].

The measurements are performed in $50 \Omega$ systems and require $\mathrm{GHz}$ technology to match the timing properties of the signals induced in SCVD sensors. $2 \mathrm{GHz}$ broadband amplifiers are used for the amplification of the detector signal.

TCT measurements require detectors and electronics to be well shielded from electromagnetic noise. The capacitance of the detector is to be kept small, in order to reduce the influence of the electronic time-constant to the signal shape.

A real-time data analysis system is desirable in neutron environments, where background reactions often dominate the spectrum. A recent technologic implementation allows the real-time analysis in mixed fields with extremely high data rates, namely up to interaction rates of $10 \mathrm{MHz}$ [8].

\section{Signals in diamond detectors}

When ionizing particles enter a diamond sensor, free charge carrier pairs of electrons $e^{-}$and holes $h^{+}$are created. When a bias field is applied to the diamond sensor, these free charge carriers drift to the respective electrode, inducing a current in the detector circuit, as shown in the equivalent circuit diagram of Fig. 1.

The Schockley-Ramo $[9,10]$ theorem describes the induced current $I$ by a moving charge $q$ in the electric field of a sensor with thickness $d$.

$$
I=q \cdot \frac{v_{d}}{d}
$$

According to the theorem, the current can be expressed in this electrostatic approximation at each moment of charge movement. The drift velocity $v_{d}$ of the free charge carriers depends on the applied electric field and differs for $e^{-}$ 


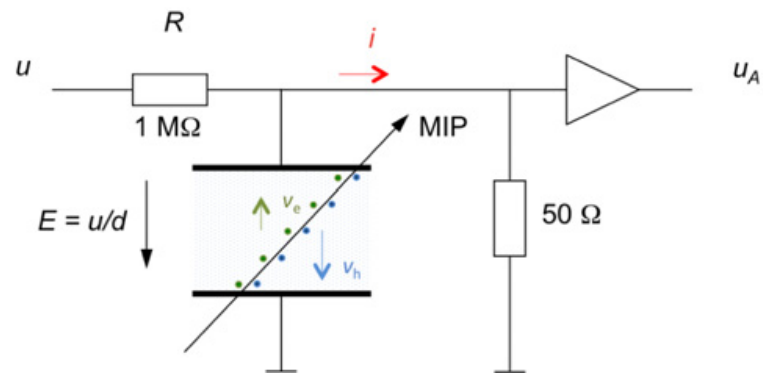

Figure 1. Equivalent circuit diagram of a SCVD diamond detector ionized by a MIP particle. The drift of $e^{-}$and $h^{+}$induce a current $i$ in the detector circuit.

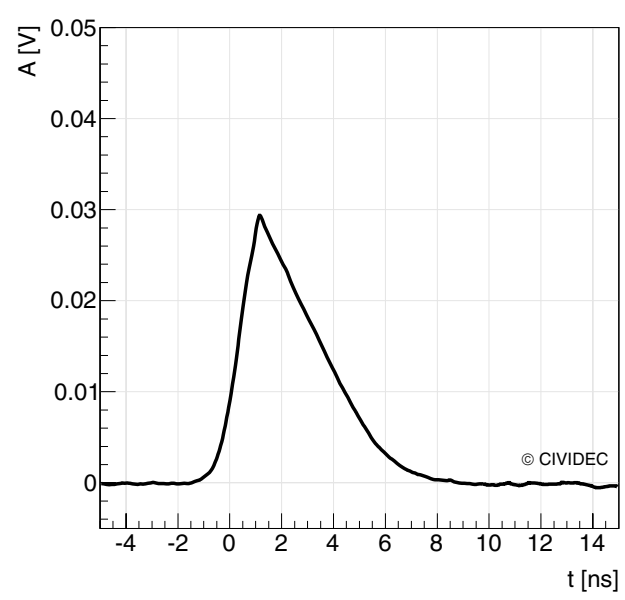

Figure 2. Average signal shape induced in a sCVD diamond sensor by MIP particles from a ${ }^{90} \mathrm{Sr}$ source.

and $h^{+}$. At typical operational conditions for diamond detectors, with $1 \mathrm{~V} / \mu \mathrm{m}$ electric field, the drift velocities are $v_{d, e^{-}}=(6.0 \pm 0.1) \cdot 10^{4} \mathrm{~m} / \mathrm{s}$ and $v_{d, h^{+}}=(8.5 \pm 0.2)$. $10^{4} \mathrm{~m} / \mathrm{s}$ for $\mathrm{e}^{-}$and $\mathrm{h}^{+}$respectively.

As the charges are moving with a constant velocity to the respective electrode, they each induce a signal of rectangular shape. The measured signal is a superposition of these rectangles induced by each moving charge. Therefore, for a homogeneous ionization track from a traversing particle, such as minimum ionizing particles (MIP), the signal shape is triangular, as shown in Fig. 2, due to the permanent absorption of free charge carriers at the electrodes. This signal shape is also seen for photon interactions in SCVD diamond sensors.

On the contrary, point-like ionizations at various locations in the sensor induce step-like signals, which are dominated by the drift of either of the charge carrier types. If the point-like ionization happens in the ballistic center of the sensor, from which the drift times for $e^{-}$and $h^{+}$are equal, the signal shape is rectangular. In this case, the drift time of charge carriers $t_{d, b c}$ is given by the relation:

$$
t_{d, b c}=\frac{d}{v_{d, h^{+}}+v_{d, e^{-}}}
$$

This is the shortest possible $t_{d}$ in a diamond sensor of a given thickness $d$ and the corresponding signal shape is therefore narrowest at the base. At the same time, when signals of the same area are compared, the signals created in the ballistic central region have the highest amplitude.

Some examples for point-like ionizations in different locations of a diamond sensor are indicated in Fig. 3.

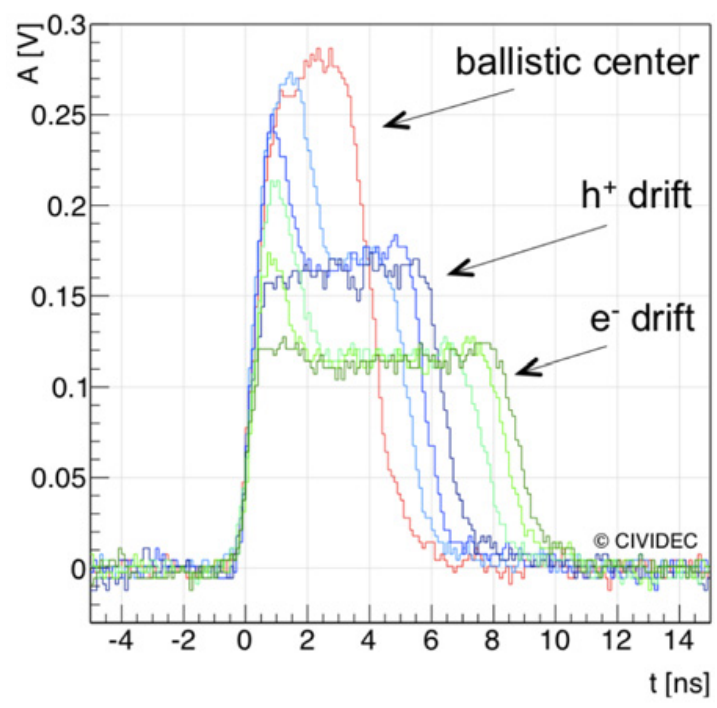

Figure 3. From Ref. [12]: Measured signals shapes in a $14.3 \mathrm{MeV}$ neutron beam. All signals correspond to a ${ }^{12} \mathrm{C}(\mathrm{n}, \alpha){ }^{9} \mathrm{Be}$ reaction in the diamond sensor.

Signals dominated by the $e^{-}$drift are indicated in green, whereas the blue signals are dominated by the $h^{+}$ drift. The rectangular signal in red is caused by a pointlike ionization at the ballistic central region (BCR), the region around the ballistic center of the sensor. These measured signals shapes, which were recorded with a sCVD sensor in a $14.3 \mathrm{MeV}$ neutron beam, were confirmed by a PSpice ${ }^{\circledR}[11]$ simulation of the experimental setup [12].

It should be noted that measured signal shapes from point-like ionizations close to the electrodes appear to be rectangular in the experiment, as is the case for the signals in Fig. 3 which are fully dominated by either $e^{-}$or $h^{+}$drift. This is due to the RC time constant of the detector circuit, which prevents to identify the very short contribution of the charge carriers which are absorbed shortly after the ionization at the near electrode.

\subsection{Signal analysis}

In the analysis of measured signals the following parameters are extracted to identify different signal shapes:

1. The amplitude of the signal $h$.

2. The full width at half maximum FWHM.

3. The width at $10 \%$ of the maximum $w_{b}$, which is a robust representative of the drift time of free charge carriers in the sensor.

4. The area $A$ of the signal, which is in SCVD diamond sensors directly proportional to the deposited energy in the detector.

In Fig. 4 these parameters are indicated on a typical rectangular signal of a sCVD diamond sensor under $\alpha$ irradiation.

The signal shape can be identified using a form factor $F$, which compares the measured area of the signal with the calculated area, under the assumption that the signal is of rectangular shape.

$$
F=\frac{\text { calculated area }}{\text { measured area }}=\frac{h \cdot w_{b}}{A},
$$




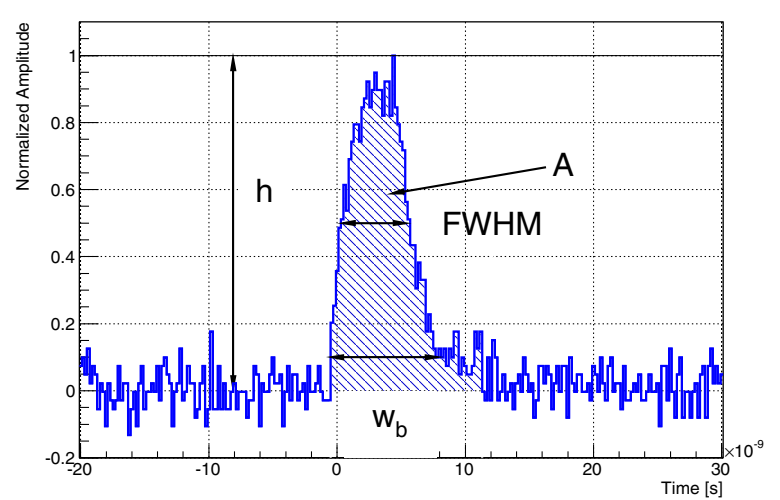

Figure 4. Rectangular signal recorded during $\alpha$ calibration in a sCVD diamond detector. The area $A$ of the signal is equivalent to the deposited energy in the detector.

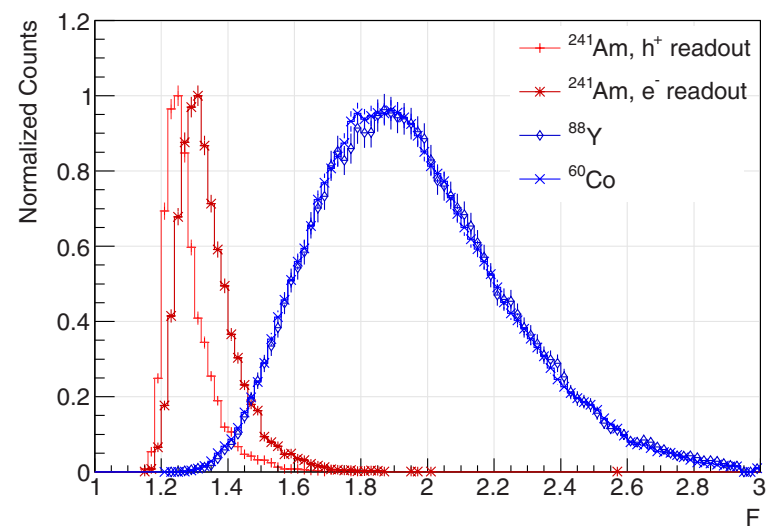

Figure 5. From Ref. [12]: Form factor $F$ for signals recorded with a sCVD diamond sensor under $\alpha$ and $\gamma$ irradiation with radioactive sources.

Given perfect geometrical shapes, $F=1$ for rectangles and $F=2$ for triangles. Due to the diffusion of the charge clouds, the RC time constant of the detector circuit and the electronic noise in the measurement, the values in the experiment diverge from these strictly theoretical values.

$F$-spectra are shown in Fig. 5 for a sCVD diamond detector under $\alpha$-irradiation from ${ }^{241} \mathrm{Am}$ and $\gamma$-irradiation from ${ }^{88} \mathrm{Y}$ and ${ }^{60} \mathrm{Co}$. The distributions are well separated and imply that $F$ can be used as indicator for the signal shape.

\subsection{Particle identification}

The different signal shapes in SCVD diamond sensors allow to deduce the interaction of the ionizing particle with the diamond sensor and consequently separate neutron reactions from other interactions and finally successfully reject background from measurements in neutron beams. Depending on the application, either of the signal parameters FWHM, $w_{b}$ and $F$, or a combination of these, is used to distinguish between neutrons and background, as described in the following.

\subsubsection{Heavy ionizing particles}

Heavy ionizing particles, like $\alpha$, tritons and fission fragments, which lead to a point-like ionization close to one electrode, are identified by the rectangular signal shape in the experiment. The width of the signal indicates from which side the particle was entering the diamond sensor.

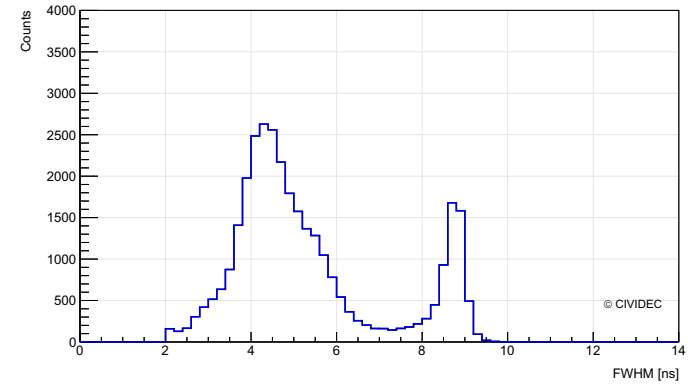

Figure 6. Measured FWHM spectrum of signals recorded in a thermal neutron beam with a $500 \mu \mathrm{m}$ thick sCVD diamond sensor with a ${ }^{6} \mathrm{Li}$ converter foil mounted close to the cathode. The signals coming from the reaction products of ${ }^{6} \mathrm{Li}(\mathrm{n}, \alpha)^{3} \mathrm{H}$ can be identified with FWHM $>7 \mathrm{~ns}$.

If the ionization happens close to the cathode, the signal is widest. For a $500 \mu \mathrm{m}$ thick sCVD diamond sensor, operated at $1 \mathrm{~V} / \mu \mathrm{m}$ the signal has a full width at half maximum of FWHM $=9 \mathrm{~ns}$, whereas an ionization close to the anode leads to signals with FWHM $=6 \mathrm{~ns}$.

For measurements in thermal neutron fields, where neutron converters yielding heavy charged particles, such as ${ }^{6} \mathrm{Li},{ }^{10} \mathrm{~B}$ or ${ }^{235} \mathrm{U}$, are used, the neutron interaction is identified by the signal induced by the heavy reaction product. In addition to the area, which is equivalent to the deposited energy in the SCVD diamond sensor, the FWHM of the signal can be used to identify the origin of the signal. This is shown in Fig. 6 where the FWHM spectrum of a measurement in a thermal neutron beam is presented, for which a ${ }^{6} \mathrm{Li}$ neutron-converter was mounted close to the cathode of the SCVD diamond sensor. The reaction products of ${ }^{6} \mathrm{Li}(\mathrm{n}, \alpha)^{3} \mathrm{H}$ have $\mathrm{FWHM}>7 \mathrm{~ns}$ and can be separated from the $\gamma$-background with smaller FWHM. In environments where only thermal neutrons or photons and MIP particles are contributing to the spectrum, the FWHM of the signals is sufficient as indicator for the current signal shape and hence for the particle identification.

\subsubsection{Traversing particles}

Traversing particles, such as MIP, photons and highenergetic protons, are identified by the triangular signal shape. Especially in thermal neutron measurements these signals are often dominating the spectrum, due to the high $\gamma$-background at nuclear reactors.

The spectrum of deposited energy of such particles in the detector is typically Landau-shaped with an exponential tail and can reach several $\mathrm{MeV}$ for $\gamma$-rays from nuclear reactions. The detection efficiency of neutrons and $\gamma$ in diamond detectors is strongly energy dependent. However, in thermal neutron beams, where an external neutron converter is used, the efficiency is approximately of the same order of magnitude for thermal neutrons and $\gamma$-rays. If the $\gamma$-flux is significantly higher than the neutron flux, the reaction products from the neutron converter are typically masked in the deposited energy spectrum by the $\gamma$-rays. In contrary, the classification by signal shape allows to separate the neutrons from $\gamma$.

\subsubsection{High-energy neutrons}

High-energy neutrons undergo nuclear reactions with ${ }^{12} \mathrm{C}$ and ${ }^{13} \mathrm{C}$ inside the diamond sensor. Apart from the elastic 


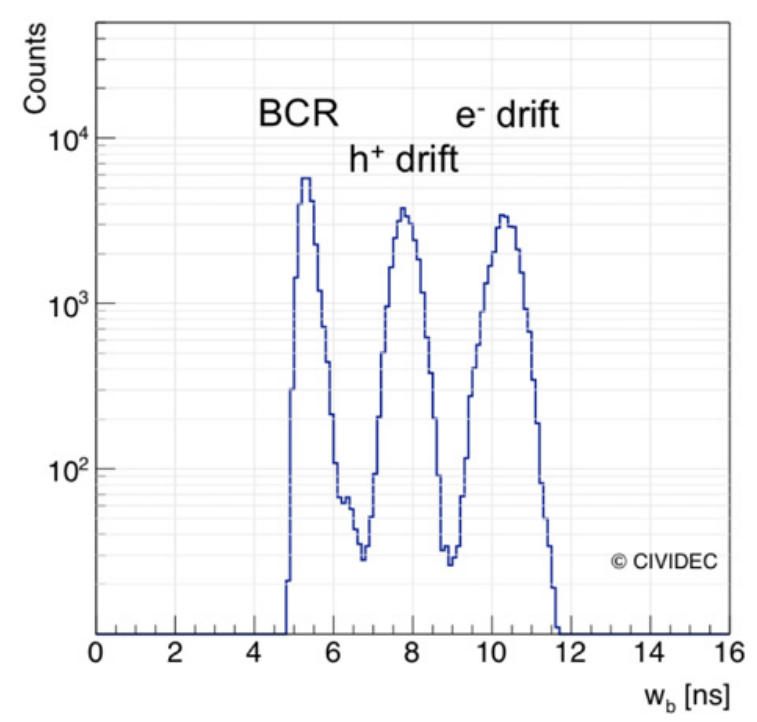

Figure 7. Base width spectrum of rectangular signals measured in a $14.3 \mathrm{MeV}$ neutron beam. $F<1.4$ was used as condition for the form factor to extract this spectrum from the total $w_{b}$ spectrum.

scattering, all reactions are threshold reactions and yield charged particles with energies $E=E_{n}+Q$, where $E_{n}$ is the energy of the incident neutron and $Q$ the reaction Q-value. All nuclear reactions are yielding heavy charged particles which ionize the detector point-like around the interaction location. In this case step-like and rectangular signals are induced when the interaction happens at a random place in the sensor or in the ballistic central region respectively.

In high-energy neutron fields all surrounding materials are the main cause of background. Neutron induced nuclear reactions yielding charged particles can dominate the recorded spectrum and mask the neutron interactions with the Carbon isotopes of the detector. This is especially crucial when Hydrogen-loaded materials are close to the sensor which produce proton recoils, which reach energies close to the energy of the incident neutron.

To reject the background coming from outside the detector, the ballistic central region is especially interesting for measurements in high-energy neutron beams. Only the neutron interaction with ${ }^{12} \mathrm{C}$ and ${ }^{13} \mathrm{C}$ in this region can induce a narrow, rectangular signal in such experiments.

The interaction location for rectangular signals can be identified via the base width $w_{b}$ of the signal as it represents the drift time of the free charge carriers. In Fig. 7 the $w_{b}$ spectrum of rectangular signals recorded in a highenergy neutron beam is shown. To extract this spectrum from the total spectrum, a qualifier of $F<1.4$ was used to extract the rectangular signals. The interaction location can clearly be distinguished for interactions in the ballistic central region, close to the anode and close to the cathode respectively via the increasing $w_{b}$ of the signals.

\section{Applications}

In the following some applications for the presented analysis technique in different neutron environments are presented.

\subsection{Thermal neutron beam}

In Ref. [13] a measurement in a thermal neutron beam with a sCVD diamond sensor is reported on. A ${ }^{6} \mathrm{Li}$

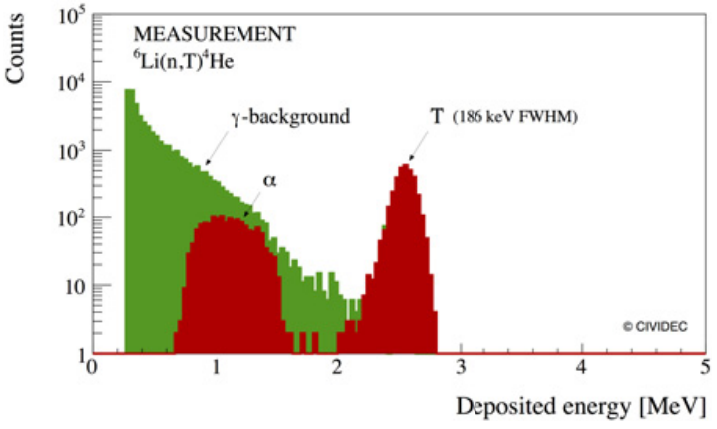

Figure 8. From Ref. [13]: Spectrum of deposited energy in a thermal neutron beam with a ${ }^{6} \mathrm{Li}$ neutron converter mounted in front of the sCVD diamond detector. The conventional spectroscopy (green) does not allow to distinguish the $\alpha$ particles. With a qualifier of $F W H M>7.5 \mathrm{~ns}$, both reaction products of ${ }^{6} \mathrm{Li}(\mathrm{n}, \alpha){ }^{3} \mathrm{H}$ were identified.

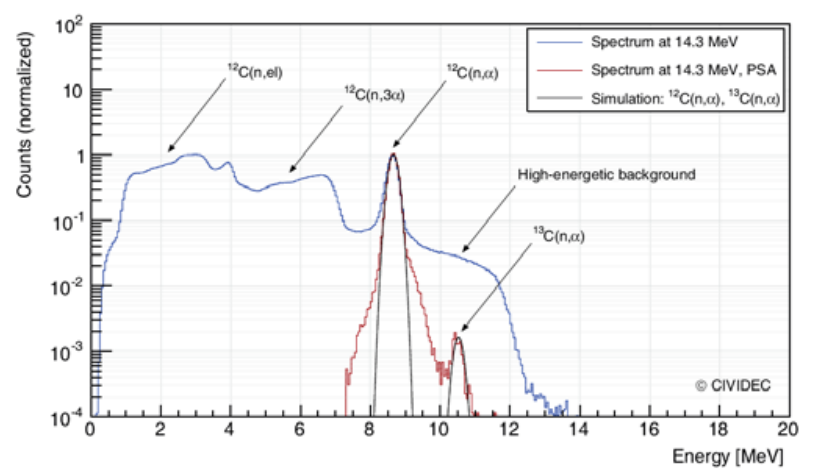

Figure 9. From Ref. [14]: Deposited energy spectrum in a sCVD diamond sensor operated in a $14.3 \mathrm{MeV}$ neutron beam (blue). The restriction to rectangular signals from the ballistic central region (red) allowed to extract the signals from ${ }^{13} \mathrm{C}(\mathrm{n}, \alpha){ }^{10} \mathrm{Be}$ from the background.

neutron converter, which was mounted close to the cathode of the detector, was used for the detection of thermal neutrons. The contribution of the reaction products of ${ }^{6} \mathrm{Li}(\mathrm{n}, \alpha)^{3} \mathrm{H}$ to the spectrum of deposited energy in the detector was masked by the high $\gamma$-background in the reactor environment. Using conventional spectroscopic electronics did not allow to identify the $\alpha$ particles in the spectrum. Only the analysis of the ionization signals from the detector and their classification by the FWHM allowed to identify their contribution, as shown in Fig. 8.

\subsection{Fast neutron beam}

An experiment in a fast neutron beam at EC-JRC Geel, Belgium, to measure the cross-section of ${ }^{13} \mathrm{C}(\mathrm{n}, \alpha){ }^{10} \mathrm{Be}$ with SCVD diamond sensors is presented in Ref. [14]. In the experimental setup Hydrogen-loaded materials were mounted in front of the detector. This caused a large background of proton recoils to mask the trace of the reaction of interest. The qualification of the detector signals using $F$ and $b_{w}$ in order to extract rectangular signals from the ballistic central region of the diamond sensor allowed to reject this background and successfully measure the cross-section of ${ }^{13} \mathrm{C}(\mathrm{n}, \alpha){ }^{10} \mathrm{Be}$. Only the restriction to the ballistic central region permitted the measurement of the reaction in question, limiting the efficiency in the experiment to $20 \%$. 


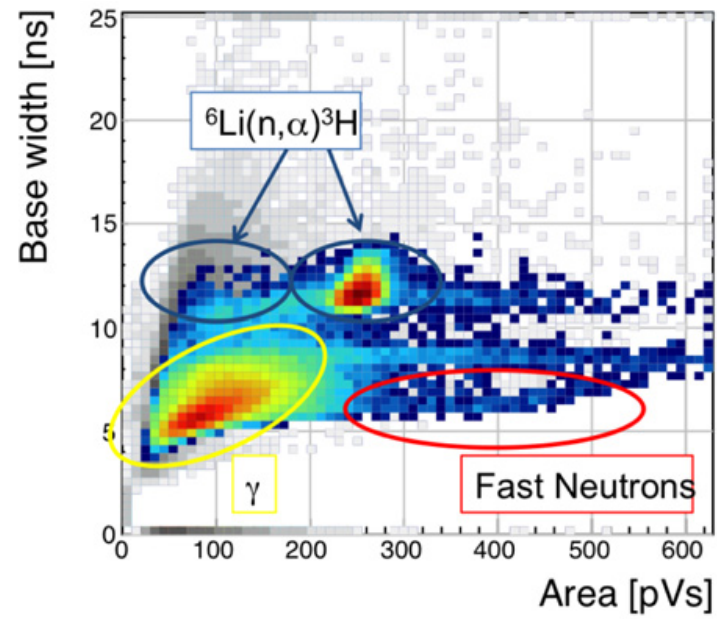

Figure 10. From Ref. [15]: Base width versus area (equivalent to deposited energy) from signals recorded in the core of a thermal nuclear reactor, where ${ }^{6} \mathrm{Li}$ was used as neutron converter. The contribution of fast neutrons to the spectrum can be distinguished from $\gamma$ and from signals from ${ }^{6} \mathrm{Li}(\mathrm{n}, \alpha)^{3} \mathrm{H}$, which indicate thermal neutrons.

\subsection{Mixed neutron field}

The presented analysis technique was used for measurements at the CROCUS reactor at EPFL, Lausanne/Switzerland, to understand whether sCVD diamond detectors can be used to discriminate $\gamma$, thermal and fast neutrons in the mixed field of a nuclear reactor. The measurement was performed with a real-time, FPGA based read-out system, as reported in Ref [15]. In this measurement a $500 \mu \mathrm{m}$ thick sCVD diamond detector with a ${ }^{6} \mathrm{Li}$ foil mounted in front of the cathode was used. The detector was inserted in the reactor core to measure in the mixed field.

In Fig. 10 the spectrum of $w_{b}$ versus the area of the recorded signals is shown. The count rate is encoded in color in a logarithmic scale. High count rates are marked in red, whereas low counts are marked in blue.

The classification of the signals by $w_{b}$ allows to clearly distinguish between the contribution of the reaction products from ${ }^{6} \mathrm{Li}(\mathrm{n}, \alpha)^{3} \mathrm{H}$, which indicate thermal neutrons and the dominating $\gamma$ field. The bias voltage of the detector was set to $0.6 \mathrm{~V} / \mu \mathrm{m}$, which explains the higher drift times than previously mentioned for $1 \mathrm{~V} / \mu \mathrm{m}$ electric field. The signals with $A>300 \mathrm{pVs}$ and $5 \mathrm{~ns}<$ $w_{b}<7 \mathrm{~ns}$ reveal the contribution of fast neutrons to the total spectrum.

Using conventional spectroscopic electronics allows only to measure the projection of the spectrum on the $\mathrm{x}$-axis and the contribution of $\gamma$ and ${ }^{3} \mathrm{H}$ from the ${ }^{6} \mathrm{Li}$ converter clearly dominate the spectrum. The $\alpha$ contribution and especially the contribution of fast neutrons in the reactor would not be distinguishable.

The presented results are preliminary and the measurements will continue, as part of the future experimental program at CROCUS [16].

\section{Conclusion}

A novel analysis technique for measurements with sCVD diamond detectors in neutron fields is presented in this paper. The intrinsic electronic properties of this sensor material are used to deduce the interaction of the ionizing particles with the diamond. This allows to identify different particle radiation, separate their contribution in the total recorded spectrum of deposited energy in the detector and finally reject background reactions from measurements in neutron fields.

Three applications of this technique are presented: In a thermal neutron beam, the photon background could successfully be rejected, which allowed to extract the thermal neutrons from the spectrum [13].

For a measurement in a high-energy neutron beam the analysis technique permitted to measure the ${ }^{13} \mathrm{C}(\mathrm{n}, \alpha)^{10} \mathrm{Be}$ cross-section, although this reaction was completely masked by proton recoils in the total recorded spectrum. The background was rejected with $99.95 \%$ efficiency, reducing the count rate of ${ }^{13} \mathrm{C}(\mathrm{n}, \alpha){ }^{10} \mathrm{Be}$ to $21.7 \%$ $[12,14]$. The analysis technique was as well used for an experiment in the core of a nuclear reactor, where it was investigated whether fast neutrons could be distinguished from thermal neutrons and $\gamma$-rays using sCVD diamond sensors. The preliminary results show that it is indeed possible to separate the different particle types from each other in the mixed field. These in-core measurements with SCVD diamond sensors will stay part of an ongoing research program [16] and shall lead to challenging future applications of the technique in reactor technology.

The authors greatly acknowledge the contributions of P.J. Bryant, M. Cerv; the team of the TRIGA Mark-II reactor at the Atomic Institute of the Vienna University of Technology, Vienna, Austria: E. Jericha, E. Klapfer, H. Schachner; the team of the Van de Graaff accelerator at EC-JRC Geel, Belgium: F. Belloni, W. Geerts, A. Plompen, P. Schillebeeckx; the CROCUS team at EPFL Lausanne, Switzerland: P. Frajtag, M. Hursin, V.P. Lamirand.

\section{References}

[1] R.S. Sussmann, CVD diamond for electronic devices and sensors (Wiley, 2009) 257-274

[2] P. Bergonzo et al., NIMA 476, 694-700 (2002)

[3] M. Pillon et al., NIMA 640, 185-191 (2011)

[4] E. Griesmayer et al., Int. Conf. on Research Reactors (2015)

[5] M. Pomorski et al., Phys. Stat. Sol. 203(12), 31523160 (2006)

[6] www.E6.com

[7] H. Pernegger et al., J. Appl. Phys. 97, 073704 (2005)

[8] www.cividec.at

[9] W. Shockley, J. Appl. Phys. 9, 635-636 (1938)

[10] S. Ramo, Proc. I.R.E. 27, 584-585 (1939)

[11] www.orcad.com

[12] C. Weiss et al., Eur. Phys. J. A 52, 269 (2016)

[13] P. Kavrigin et al., NIMA 795, 88-91 (2015)

[14] P. Kavrigin et al., Eur. Phys. J. A 52, 179 (2016)

[15] M. Cerv, Ph.D. thesis, Vienna University of Technology (under preparation)

[16] https://infoscience.epfl.ch/record/218310

[17] H. Frais-Kölbl et al., IEEE 51, 6 (2004) 